\title{
GALOIS POINTS FOR DOUBLE-FROBENIUS NONCLASSICAL CURVES
}

\author{
HERIVELTO BORGES AND SATORU FUKASAWA
}

\begin{abstract}
We determine the distribution of Galois points for plane curves over a finite field of $q$ elements, which are Frobenius nonclassical for different powers of $q$. This family is an important class of plane curves with many remarkable properties. It contains the Dickson-Guralnick-Zieve curve, which has been recently studied by Giulietti, Korchmáros, and Timpanella from several points of view. A problem posed by the second author in the theory of Galois points is modified.
\end{abstract}

\section{INTRODUCTION}

Let $\mathbb{F}_{q}$ be a finite field with $q \geq 2$, and let $\mathcal{F} \subset \mathbb{P}^{2}$ be the plane curve defined by $F(x, y, z)=D_{1}(x, y, z) / D_{2}(x, y, z)$, where

$$
D_{1}=\left|\begin{array}{ccc}
x & x^{q^{m}} & x^{q^{n}} \\
y & y^{q^{m}} & y^{q^{n}} \\
z & z^{q^{m}} & z^{q^{n}}
\end{array}\right| \text { and } D_{2}=\left|\begin{array}{ccc}
x & x^{q} & x^{q^{2}} \\
y & y^{q} & y^{q^{2}} \\
z & z^{q} & z^{q^{2}}
\end{array}\right| \text {, }
$$

and $n$ and $m$ are coprime. According to [1, p.544 and Theorem 3.4], $F$ is a homogeneous polynomial of degree $q^{n}+q^{m}-q^{2}-q$ over $\mathbb{F}_{q}$, which is irreducible over the algebraic closure $\overline{\mathbb{F}}_{q}$. In 2009, the first author characterized these curves as the unique double-Frobenius nonclassical plane curves for different powers $q^{n}$ and $q^{m}$, with $\operatorname{gcd}(n, m)=1$. Other significant features, such as a large number of $\mathbb{F}_{q^{n}}$-rational points (meeting the Stöhr-Voloch bound) and the arc property in the case $m=1$ were also noted [1. This important family of curves contains the newly coined Dickson-Guralnick-Zieve (DGZ) curve: case $(n, m)=(3,1)$. Additional remarkable properties of the DGZ curve, such as a large automorphism group and positive $p$-rank, have been recently proved by Giulietti, Korchmáros, and Timpanella [6].

2010 Mathematics Subject Classification. 14H50, 11G20.

Key words and phrases. Galois point, Frobenius nonclassical curve, rational point. The first author was partially supported by FAPESP grant 2017/04681-3.

The second author was partially supported by JSPS KAKENHI Grant Number 16K05088. 
In this article, we consider Galois points for the curves $\mathcal{F}$ over $\overline{\mathbb{F}}_{q}$ (see [7, 9] for the definition of Galois point). The set of all Galois points for the plane curve $\mathcal{F}$ on the projective plane is denoted by $\Delta(\mathcal{F})$.

Our main result is the following.

Theorem 1. Let $n \geq 3$ and $m \geq 1$ be integers such that $n>m$ and $\operatorname{gcd}(n, m)=1$. For the $\left(q^{n}, q^{m}\right)$-Frobenius nonclassical curve $\mathcal{F} \subset \mathbb{P}^{2}$,

$$
\Delta(\mathcal{F})=\emptyset \text { or } \mathbb{P}^{2}\left(\mathbb{F}_{q}\right)
$$

The latter case occurs if and only if $(n, m)=(3,1)$ or $(3,2)$.

Since the result for the case where $(n, m)=(3,1)$ or $(3,2)$ gives a negative answer to the problem [3. Problem 1], posed by the second author, it is modified as follows.

Problem 1. Let $\mathcal{C}$ be a plane curve over $\mathbb{F}_{q}$. Assume that $\Delta(\mathcal{C})=\mathbb{P}^{2}\left(\mathbb{F}_{q}\right)$. Then, is it true that $\mathcal{C}$ is projectively equivalent to the Hermitian, Ballico-Hefez or the $\left(q^{n}, q^{m}\right)$-Frobenius nonclassical curve of type $(n, m)=(3,1)$ or $(3,2)$ ? Or, more basically, is $\mathcal{C}$ Frobenius nonclassical?

The result in [4] is contained in this article.

\section{Preliminaries}

Let $\mathcal{C} \subset \mathbb{P}^{2}$ be an irreducible plane curve of degree $d$, and let $r: \hat{\mathcal{C}} \rightarrow \mathcal{C}$ be the normalization. For different points $P$ and $Q \in \mathbb{P}^{2}$, the line passing through $P$ and $Q$ is denoted by $\overline{P Q}$. For a point $P \in \mathbb{P}^{2}, \pi_{P}: \mathcal{C} \rightarrow \mathbb{P}^{1}$ represents the projection from $P$. The composite map $\pi_{P} \circ r: \hat{\mathcal{C}} \rightarrow \mathbb{P}^{1}$ is denoted by $\hat{\pi}_{P}$. The ramification index at $\hat{Q} \in \hat{\mathcal{C}}$ is represented by $e_{\hat{Q}}$. When $r^{-1}(Q)$ consists of a unique point $\hat{Q} \in \hat{\mathcal{C}}$, the index $e_{\hat{Q}}$ is denoted also by $e_{Q}$.

Fact 1. For the projection $\hat{\pi}_{P}$, the following holds.

(a) For each point $\hat{Q} \in \hat{C}$ with $Q=r(\hat{Q}) \neq P$, it follows that $e_{\hat{Q}}=\operatorname{ord}_{\hat{Q}} h_{P Q}$, where $h_{P Q}$ is a linear polynomial defining the line $\overline{P Q}$.

(b) If $P \in \mathcal{C} \backslash \operatorname{Sing}(\mathcal{C})$, then $e_{P}=\operatorname{ord}_{P} h_{P}-1$, where $h_{P}$ is a linear polynomial defining the tangent line at $P$.

(c) If $P$ is an ordinary singularity of $\mathcal{C}$ with multiplicity $m(P)$, that is, $P$ has $m(P)$ tangent lines, then for each tangent line defined by $h=0$ at $P$, there exists a unique point $\hat{P} \in r^{-1}(P)$ such that $e_{\hat{P}}=\operatorname{ord}_{\hat{P}} h-1$.

According to [1, Proposition 3.2], the following holds.

Fact 2. Let $\mathcal{F} \subset \mathbb{P}^{2}$ be the $\left(q^{n}, q^{m}\right)$-Frobenius nonclassical curve, and let $S \subset \mathbb{P}^{2}$ be the set of all points $R$ such that $R$ is contained in some $\mathbb{F}_{q}$-line. 
(a) For the case where $m>1, \operatorname{Sing}(\mathcal{F})=\mathbb{P}^{2}\left(\mathbb{F}_{q^{n-m}}\right)$. Let $Q=r(\hat{Q}) \in \operatorname{Sing}(\mathcal{F})$ be of multiplicity $m(Q)$.

(i) If $Q \notin S$, then $m(Q)=q^{m}, r^{-1}(Q)=\{\hat{Q}\}$, and there exists a unique line $T_{Q} \ni Q$ such that $\operatorname{ord}_{\hat{Q}} T_{Q}=q^{m}+1$.

(ii) If $Q \in S$ and $Q \notin \mathbb{P}^{2}\left(\mathbb{F}_{q}\right)$, then $m(Q)=q^{m}-1, r^{-1}(Q)=\{\hat{Q}\}$, and there exists a unique line $T_{Q} \ni Q$ such that $\operatorname{ord}_{\hat{Q}} T_{Q}=q^{m}$.

(iii) If $Q \in \mathbb{P}^{2}\left(\mathbb{F}_{q}\right)$, then $m(Q)=q^{m}-q, r^{-1}(Q)$ consists of exactly $m(Q)$ points, and $Q$ is an ordinary singularity. For each tangent line $T$ at $Q$, the intersection multiplicity of $\mathcal{F}$ and $T$ at $Q$ is equal to $q^{n}-q$.

(b) For the case where $m=1$ and $q>2$, Sing $(\mathcal{F})=\mathbb{P}^{2}\left(\mathbb{F}_{q^{n-1}}\right) \backslash \mathbb{P}^{2}\left(\mathbb{F}_{q}\right)$. Let $Q=r(\hat{Q}) \in \operatorname{Sing}(\mathcal{F})$ be of multiplicity $m(Q)$.

(i) If $Q \notin S$, then $m(Q)=q, r^{-1}(Q)=\{\hat{Q}\}$, and there exists a unique line $T_{Q} \ni Q$ such that $\operatorname{ord}_{\hat{Q}} T_{Q}=q+1$.

(ii) If $Q \in S$ and $Q \notin \mathbb{P}^{2}\left(\mathbb{F}_{q}\right)$, then $m(Q)=q-1, r^{-1}(Q)=\{\hat{Q}\}$, and there exists a unique line $T_{Q} \ni Q$ such that $\operatorname{ord}_{\hat{Q}} T_{Q}=q$.

(c) For the case where $(m, q)=(1,2)$, $\operatorname{Sing}(\mathcal{F})=\mathbb{P}^{2}\left(\mathbb{F}_{2^{n-1}}\right) \backslash S$. If $Q=r(\hat{Q}) \in$ $\operatorname{Sing}(\mathcal{F})$ with multiplicity $m(Q)$, then $m(Q)=2, r^{-1}(Q)=\{\hat{Q}\}$, and there exists a unique line $T_{Q} \ni Q$ such that $\operatorname{ord}_{\hat{Q}} T_{Q}=3$.

If $P$ is a Galois point, then the following holds (see [8, III.7.2]).

Fact 3. If the projection $\hat{\pi}_{P}: \hat{\mathcal{C}} \rightarrow \mathbb{P}^{1}$ is a Galois covering, then we have the following.

(a) If $\hat{Q}_{1}$ and $\hat{Q}_{2} \in \hat{\mathcal{C}}$ have the same image, then $e_{\hat{Q}_{1}}=e_{\hat{Q}_{2}}$.

(b) For each point $\hat{Q} \in \hat{\mathcal{C}}$, the index $e_{\hat{Q}}$ divides the degree $\operatorname{deg} \hat{\pi}_{P}$.

Combining Facts 1(a), 2 and 3(a), we have the following.

Corollary 1. Let $\hat{\pi}_{P}: \hat{\mathcal{F}} \rightarrow \mathbb{P}^{1}$ be the projection, and let $Q=r(\hat{Q}) \in \mathcal{F} \backslash\{P\}$ be a singular point.

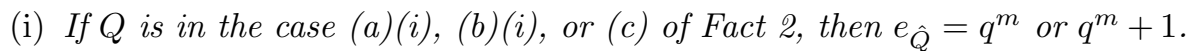

(ii) If $Q$ is in the case (a)(ii) or (b)(ii) of Fact Q, then $e_{\hat{Q}}=q^{m}-1$ or $q^{m}$.

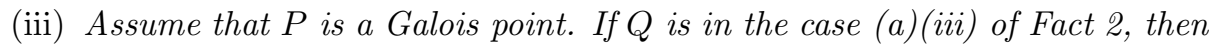
$e_{\hat{Q}}=1$.

\section{Frobenius nonclassicality and Galois points}

Proposition 1. Let $\mathcal{C} \subset \mathbb{P}^{2}$ be a q-Frobenius nonclassical curve over $\mathbb{F}_{q}$. Assume that $P$ is a Galois point for $\mathcal{C}$ and $Q \in \mathcal{C} \backslash \operatorname{Sing}(\mathcal{C})$ is a ramification point for the projection from $P$. 
(a) If $P \in\left(\mathbb{P}^{2} \backslash \mathcal{C}\right) \cup \operatorname{Sing}(\mathcal{C})$, then the line $\overline{P Q}$ is defined over $\mathbb{F}_{q}$.

(b) If $P=Q$, then the tangent line $T_{P} \mathcal{C}$ at $P$ is defined over $\mathbb{F}_{q}$.

(c) If $P \in \mathcal{C} \backslash \operatorname{Sing}(\mathcal{C})$ and there exists a point $Q^{\prime} \in(\mathcal{C} \backslash \operatorname{Sing}(\mathcal{C})) \cap(\overline{P Q} \backslash\{P, Q\})$, then the line $\overline{P Q}$ is defined over $\mathbb{F}_{q}$.

Proof. We prove assertions (a) and (c). Let $Q^{q}$ and $L^{q}$ be the $q$-Frobenius images of the point $Q$ and the line $L:=\overline{P Q}$, respectively. Considering Fact 3 (a), we need only prove the claim under the assumption that $Q^{q} \neq P$. Since $Q$ is a ramification point, it follows from Fact 1 (a) that the line $L$ is tangent to $\mathcal{C}$ at $Q$. It follows that $L^{q}$ is tangent to $\mathcal{C}$ at $Q^{q}$. Now the $q$-Frobenius nonclassicality of $\mathcal{C}$ implies that $Q^{q}$ lies on $L$. The assumption $Q^{q} \neq P$ and Fact 3(a) imply that $L$ is tangent to $\mathcal{C}$ at $Q^{q}$. Hence $L=L^{q}$, that is, $L$ is an $\mathbb{F}_{q}$-line.

We consider the case where $P$ is a ramification point of $\hat{\pi}_{P}$. By the Frobenius nonclassicality, $P^{q} \in T_{P} \mathcal{C}$. If $P^{q} \neq P$, then Fact 3(a) implies that $P^{q}$ is a ramification point. Therefore, the tangent line $T_{P^{q}} \mathcal{C}$ is the same as $T_{P} \mathcal{C}$. Similar to the above proof, $T_{P} \mathcal{C}$ is $\mathbb{F}_{q}$-rational.

Corollary 2. Let $\mathcal{F} \subset \mathbb{P}^{2}$ be the $\left(q^{n}, q^{m}\right)$-Frobenius nonclassical curve over $\mathbb{F}_{q}$. Assume that $P$ is a Galois point for $\mathcal{F}$ and $Q \in \mathcal{F} \backslash \operatorname{Sing}(\mathcal{F})$ is a ramification point for the projection from $P$.

(a) If $P \in\left(\mathbb{P}^{2} \backslash \mathcal{F}\right) \cup \operatorname{Sing}(\mathcal{F})$, then the line $\overline{P Q}$ is defined over $\mathbb{F}_{q}$.

(b) If $P=Q$, then the tangent line $T_{P} \mathcal{F}$ at $P$ is defined over $\mathbb{F}_{q}$.

(c) If $P \in \mathcal{F} \backslash \operatorname{Sing}(\mathcal{F})$ and there exists a point $Q^{\prime} \in(\mathcal{F} \backslash \operatorname{Sing}(\mathcal{F})) \cap(\overline{P Q} \backslash$ $\{P, Q\})$, then the line $\overline{P Q}$ is defined over $\mathbb{F}_{q}$.

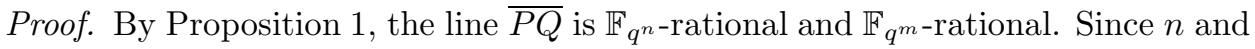
$m$ are coprime, the line is defined over $\mathbb{F}_{q}$.

\section{InNER SMOOTH GaLOIS POINTS}

Let $P \in \mathcal{F} \backslash \operatorname{Sing}(\mathcal{F})$ be an inner Galois point. It follows from Fact 1 (b) that $e_{P}=I_{P}\left(\mathcal{F}, T_{P} \mathcal{F}\right)-1$ for the projection $\hat{\pi}_{P}$, where $I_{P}\left(\mathcal{F}, T_{P} \mathcal{F}\right)$ is the intersection multiplicity of the curve $\mathcal{F}$ and the tangent line $T_{P} \mathcal{F}$ at $P$. Assume that $(m, q) \neq$ $(1,2)$. Then $I_{P}\left(\mathcal{F}, T_{P} \mathcal{F}\right) \geq q^{m} \geq 3$ ([1, Theorem 2.6]), and hence $P$ is a ramification point. By Corollary 2 (b), the tangent line $T_{P} \mathcal{F}$ is $\mathbb{F}_{q}$-rational. If $m>1$, then there exists an ordinary singularity on $T_{P} \mathcal{F}$ (Fact 2 (a)), by Corollary 1 (iii), and this is a contradiction to Fact 3(a). If $m=1$ and $q>2$, then $T_{P} \mathcal{F}$ contains a singular point $Q$ with index $e_{Q}=q$ or $q-1$ (Corollary 1(ii)). It follows from Fact 3(b) that $e_{Q}$ divides $\operatorname{deg} \hat{\pi}_{P}=q^{n}-q^{2}-1$. This is impossible. 
Assume that $(m, q)=(1,2)$ and $n>3$. Since $n-m>2$, there exists a singular point $Q$ such that there does not exist an $\mathbb{F}_{2}$-line containing $Q$ (Fact 2 (c)). Therefore, $\overline{P Q}$ is not an $\mathbb{F}_{2}$-line. Since $\operatorname{deg} \hat{\pi}_{P}=q^{n}-q^{2}-1$, it follows from Corollary 1 (i) and Fact 3 (b) that $e_{Q}=3$. Since the tangent line at the point $Q$ is $\mathbb{F}_{2^{n-1} \text {-rational, }}$

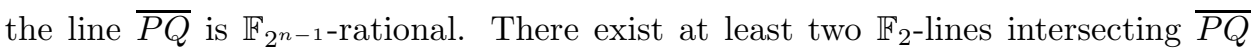
at points of $\mathcal{F}$ different from $P$. It follows from Fact 2(c) that such points are smooth points. According to Corollary 2 (c), the line $\overline{P Q}$ is $\mathbb{F}_{q}$-rational. This is a contradiction.

\section{THE CASE WHERE $n-m>2$}

Assume that $n-m>2$ and $P \in\left(\mathbb{P}^{2} \backslash \mathcal{F}\right) \cup \operatorname{Sing}(\mathcal{F})$ is a Galois point. Since $n-m>2$, there exists a singular point $Q \neq P$ not contained in any $\mathbb{F}_{q}$-line (Fact 22). Then $Q$ is a ramification point for $\hat{\pi}_{P}$ with index $e_{Q}=q^{m}$ or $q^{m}+1$ and the line $\overline{P Q}$ is not $\mathbb{F}_{q}$-rational. By Corollary 2 (a), $\overline{P Q}$ does not contain a smooth point. It follows from Fact 3 (a) and Corollary 1 that $\overline{P Q}$ is an $\mathbb{F}_{q^{n-m}}$-line and $\overline{P Q} \cap \mathcal{F}$ consists of only singular points. By considering the intersection points given by $\overline{P Q}$ and $\mathbb{F}_{q}$-lines, there exists a point $Q^{\prime} \in \overline{P Q}$ such that $Q^{\prime}$ is not $\mathbb{F}_{q^{-}}$-rational and is contained in some $\mathbb{F}_{q^{-}}$line. It follows from Proposition 1 that $e_{Q^{\prime}}=q^{m}-1$ or $q^{m}$. By Fact 3 (a), $e_{Q^{\prime}}=e_{Q}$ and hence $e_{Q^{\prime}}=e_{Q}=q^{m}$. Note that the number of points in $\overline{P Q} \cap \mathcal{F}$ is equal to $q^{n-m}+1$ or $q^{n-m}$.

Assume that $P \in \mathbb{P}^{2} \backslash \mathcal{F}$. Then $q^{n}+q^{m}-q^{2}-q=e_{Q}\left(q^{n-m}+1\right)$, or $e_{Q} q^{n-m}$. This is impossible.

Assume that $P \in \operatorname{Sing}(\mathcal{F}) \backslash \mathbb{P}^{2}\left(\mathbb{F}_{q}\right)$. Since $e_{Q}=q^{m} \operatorname{divides} \operatorname{deg} \hat{\pi}_{P}$, by Fact 2 the multiplicity $m(P)$ is equal to $q^{m}$. Then $q^{n}-q^{2}-q=e_{Q}\left(q^{n-m}+1\right), e_{Q} q^{n-m}$, or $e_{Q}\left(q^{n-m}-1\right)$. This is also impossible.

Assume that $P \in \operatorname{Sing}(\mathcal{F}) \cap \mathbb{P}^{2}\left(\mathbb{F}_{q}\right)$. By Fact $2 m>1$. It follows from Fact 2(a) that $\operatorname{deg} \hat{\pi}_{P}=q^{n}-q^{2}$ and $(\overline{P Q} \backslash\{P\}) \cap \mathcal{F}$ consists of exactly $q^{n-m}$ singular points. According to [1, Remark 3.3], $\overline{P Q}$ is a tangent line at $P$. Since $P$ is an ordinary singularity, by Fact 1(c), the fiber of the point corresponding to $\overline{P Q}$ for $\hat{\pi}_{P}$ contains exactly $q^{n-m}+1$ points. It follows that $e_{Q}\left(q^{n-m}+1\right)=q^{n}-q^{2}$. This is impossible.

\section{The CASE WHERE $n-m=2$}

Assume that $n-m=2,(m, q) \neq(1,2)$ and $P \in\left(\mathbb{P}^{2} \backslash \mathcal{F}\right) \cup \operatorname{Sing}(\mathcal{F})$ is a Galois point. Let $Q$ be a singular point different from $P$ that is contained in $\mathbb{P}^{2}\left(\mathbb{F}_{q^{2}}\right) \backslash \mathbb{P}^{2}\left(\mathbb{F}_{q}\right)$ (Fact 21). Since any $\mathbb{F}_{q^{2}}$-line contains an $\mathbb{F}_{q^{-}}$-point, $Q$ is a ramification point with index $q^{m}-1$ or $q^{m}$. 
Assume that $m>1$. It follows from Fact 3 (a) and Corollary 1(iii) that $\overline{P Q} \backslash\{P\}$ does not contain an ordinary singularity. Therefore, $\overline{P Q} \backslash\{P\}$ does not contain an $\mathbb{F}_{q}$-rational point. In particular, $\overline{P Q}$ is not $\mathbb{F}_{q}$-rational. By Corollary 2 (a), $\overline{P Q}$ does not contain a smooth point. It follows from Fact 3(a) and Corollary 1 that $\overline{P Q}$ is an $\mathbb{F}_{q^{2}}$-line. Since any $\mathbb{F}_{q^{2}}$-line contains an $\mathbb{F}_{q}$-point, the point $P$ must be an $\mathbb{F}_{q}$-point, and hence a point with multiplicity $q^{m}-q$. Then $\overline{P Q}$ is a tangent line at $P$. Furthermore, the set $(\mathcal{F} \cap \overline{P Q}) \backslash\{P\}$ consists of exactly $q^{2} \mathbb{F}_{q^{2}}$-rational but not $\mathbb{F}_{q}$-rational points. Note that there exists a unique point $\hat{P} \in r^{-1}(P)$ such that the image $\hat{\pi}_{P}(\hat{P})$ corresponds to the line $\overline{P Q}$. Therefore, the fiber $\hat{\pi}_{P}^{-1}(\overline{P Q})$ contains exactly $q^{2}+1$ points. It follows that $e_{Q}\left(q^{2}+1\right)=q^{n}-q^{2}$. This is impossible.

Assume that $m=1$. Then $n=3$. If $\overline{P Q}$ contains a smooth point, then by Corollary 2(a), $\overline{P Q}$ is $\mathbb{F}_{q}$-rational. If $\overline{P Q}$ does not contain a smooth point, then it follows from Fact 3(a) and Corollary 1 that $\overline{P Q}$ is $\mathbb{F}_{q^{2}}$-rational. Therefore, $P$ is

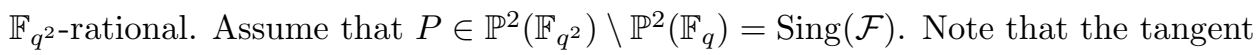
line $T$ at $P$ is defined over $\mathbb{F}_{q}$, and the tangent line at each singular point $R$ in $T \cap\left(\mathbb{P}^{2}\left(\mathbb{F}_{q^{2}}\right) \backslash \mathbb{P}^{2}\left(\mathbb{F}_{q}\right)\right)$ is the same as $T$. Then $e_{R}=q$. It follows from Fact $3(\mathrm{~b})$ that $e_{R}$ divides $\operatorname{deg} \hat{\pi}_{P}=q^{3}-q^{2}-(q-1)$. This is a contradiction.

In conclusion, it follows that if $\Delta(\mathcal{F}) \neq \emptyset$, then $m=1$ and $\Delta(\mathcal{F}) \subset \mathbb{P}^{2}\left(\mathbb{F}_{q}\right) \subset$ $\mathbb{P}^{2} \backslash \mathcal{F}$.

Remark 1. When $(n, m, q)=(3,1,2)$, the curve $\mathcal{F}$ is given by

$$
F(x, y, z)=\left(x^{2}+x z\right)^{2}+\left(x^{2}+x z\right)\left(y^{2}+y z\right)+\left(y^{2}+y z\right)^{2}+z^{4}
$$

(see [1, p.542] or [6, Remark 1]). In this case, it is known that the claim follows ([2, Theorem 4]).

\section{THE CASE WHERE $n-m=1$}

Assume that $n-m=1$ and $P \in \mathbb{P}^{2} \backslash \mathcal{F}$ is a Galois point. Since all singular points are ordinary singularities (Fact 2), by Corollary 1 (iii), the projection from $P$ is not ramified at such points. Therefore, there exists a smooth point $Q \in \mathcal{F}$ that is a ramification point. By Corollary 2 (a), the line $\overline{P Q}$ is $\mathbb{F}_{q}$-rational. However, there exists an ordinary singularity in $\overline{P Q}$. This is a contradiction to Fact 3(a).

Assume that $n-m=1, m>2$ and $P \in \operatorname{Sing}(\mathcal{F})$ is a Galois point. Let $T$ be a tangent line at $P$. By Facts 1(c) and 2(a)(iii), there exists a ramification point $\hat{P} \in r^{-1}(P)$ contained in the fiber of the point corresponding to $T$ for the projection $\hat{\pi}_{P}$. Since $m>2$, there exists a point $Q \in(\mathcal{F} \cap T) \backslash\{P\}$ (Fact 2(a)(iii)). By [1, Remark 3.3], since $T$ is not an $\mathbb{F}_{q}$-line, $Q$ is a smooth point. By Fact 3 (a), $Q$ is a 
ramification point. It follows from Corollary 2 (a) that $T$ is $\mathbb{F}_{q}$-rational. This is a contradiction.

Accordingly, it follows that if $\Delta(\mathcal{F}) \neq \emptyset$, then $m=2$ and $\Delta(\mathcal{F}) \subset \operatorname{Sing}(\mathcal{F})=$ $\mathbb{P}^{2}\left(\mathbb{F}_{q}\right)$.

\section{The CASE Where $(n, m)=(3,1)$ OR $(3,2)$}

It is easily verified that the projective linear group $P G L\left(3, \mathbb{F}_{q}\right)$ acts on $\mathcal{F}$ by the definitions of $D_{1}$ and $D_{2}$ as in (11) (see also [6, Lemma 4.1]). Therefore, the matrices

$$
\sigma_{\gamma, \beta}=\left(\begin{array}{ccc}
1 & 0 & 0 \\
\gamma & 1 & \beta \\
0 & 0 & 1
\end{array}\right) \text { and } \tau_{\mu}=\left(\begin{array}{ccc}
1 & 0 & 0 \\
0 & \mu & 0 \\
0 & 0 & 1
\end{array}\right) \in \operatorname{PGL}\left(3, \mathbb{F}_{q}\right)
$$

act on $\mathcal{F}$, where $\gamma, \beta \in \mathbb{F}_{q}$ and $\mu^{q-1}=1$. Note that a rational function $x / z$ is fixed by the actions of $\sigma_{\gamma, \beta}$ and $\tau_{\mu}$. This implies that $\pi_{P} \circ \sigma_{\gamma, \beta}=\pi_{P}$ and $\pi_{P} \circ \tau_{\mu}=\pi_{P}$, where $P=(0: 1: 0)$. Note also that $\operatorname{deg} \pi_{P}=q^{3}-q^{2}$ if and only if $(n, m)=(3,1)$ or $(3,2)$ (Fact 2). Considering the action of $P G L\left(3, \mathbb{F}_{q}\right)$, it is inferred that $\mathbb{P}^{2}\left(\mathbb{F}_{q}\right) \subset \Delta(\mathcal{F})$ holds, if $(n, m)=(3,1)$ or $(3,2)$.

Remark 2. (1) If $(n, m)=(3,1)$ or $(3,2)$, then the associated Galois group $G_{P}$ of a Galois point $P \in \Delta(\mathcal{F})$ is isomorphic to the semidirect product $\mathbb{F}_{q}^{\oplus 2} \rtimes \mathbb{F}_{q}^{*}$, where the action of $\mathbb{F}_{q}^{*}$ on $\mathbb{F}_{q}^{\oplus 2}$ is given by $(\mu,(\gamma, \beta)) \mapsto(\mu \gamma, \mu \beta)$.

(2) All points in $\mathbb{P}^{2}\left(\mathbb{F}_{q}\right)$ are quasi-Galois points which are not Galois points, if $(n, m) \neq(3,1)$ or $(3,2)$ (see [5] for the definition of quasi-Galois point).

\section{REFERENCES}

[1] H. Borges, On multi-Frobenius non-classical plane curves, Arch. Math. 93 (2009), 541-553.

[2] S. Fukasawa, On the number of Galois points for a plane curve in positive characteristic, III, Geom. Dedicata 146 (2010), 9-20.

[3] S. Fukasawa, Rational points and Galois points for a plane curve over a finite field, Finite Fields Appl. 39 (2016), 36-42.

[4] S. Fukasawa, Galois points for the Dickson-Guralnick-Zieve curve, arXiv:1806.02183, unpublished.

[5] S. Fukasawa, K. Miura and T. Takahashi, Quasi-Galois points, I: Automorphism groups of plane curves, Tohoku Math. J., to appear.

[6] M. Giulietti, G. Korchmáros and M. Timpanella, On the Dickson-Guralnick-Zieve curve, preprint, arXiv: 1805.05618 .

[7] K. Miura and H. Yoshihara, Field theory for function fields of plane quartic curves, J. Algebra 226 (2000), 283-294.

[8] H. Stichtenoth, Algebraic Function Fields and Codes, Universitext, Springer-Verlag, Berlin (1993). 
[9] H. Yoshihara, Function field theory of plane curves by dual curves, J. Algebra 239 (2001), 340-355.

Universidade de São Paulo, Inst. de Ciências Matemáticas e de Computação, São Carlos, SP 13560-970, Brazil.

E-mail address: hborges@icmc.usp.br

Department of Mathematical Sciences, Faculty of Science, Yamagata University, KoJiraKaWA-MACHi 1-4-12, Yamagata 990-8560, Japan.

E-mail address: s.fukasawa@sci.kj.yamagata-u.ac.jp 